\title{
Skill of an Aircraft Wake-Vortex Model Using Weather Prediction and Observation
}

\author{
Michael Frech* and Frank Holzäpfel ${ }^{\ddagger}$ \\ DLR, German Aerospace Center, Oberpfaffenhofen, 82383 Wessling, Germany
}

DOI: $\underline{10.2514 / 1.28983}$

\begin{abstract}
The performance of the two-phase probabilistic wake-vortex transport and decay model using numerical weather prediction and weather observations as input is analyzed using data from a wake measurement campaign carried out at Frankfurt airport during fall 2004. The wake-vortex observations include wakes evolving in and out of ground effects. The best forecast quality is achieved for wakes evolving in ground effect. We include the simplified hazardarea prediction model to compute the time to clear the corridor from hazardous wakes and to assess the potential capacity gain for single-runway operations. The highest-capacity potential can be expected when weather observations are employed for wake predictions. The limiting factor for capacity are wakes evolving in ground effect. Out of ground effect, the self-induced vertical transport of the wake vortex proves to be a robust mechanism to clear the approach corridor in an efficient way. This is found independently from the source of meteorological input. In contrast, the consideration of lateral wake transport alone leads to marginal-capacity potential. The analysis also shows that weather data along the glide path can be provided by numerical weather prediction for safe wake prediction.
\end{abstract}

\section{Nomenclature}

vortex spacing

lateral displacement

Brunt-Väisälä frequency

time

axial velocity

aircraft ground speed

axial coordinate

spanwise coordinate

vertical coordinate

circulation

eddy dissipation rate

potential temperature

standard deviation

$b$
$l$
$N$
$q$
$t$
$u$
$v$
$V$
$w$
$x$
$y$
$z$
$\Gamma$
$\epsilon$
$\theta$
$\sigma$

root-mean-square turbulence velocity

lateral velocity, vertical displacement

descent speed, vertical wind velocity

\section{Subscripts}

c $\quad=$

$l=$ time to leave the corridor

lead $=$ lead time

$0=$ initial value

Superscript

$* \quad=$

normalized by initial vortex parameters

$b_{0}, t_{0}, w_{0}$, and $\Gamma_{0}$

\section{Introduction}

A LREADY-EXISTING and expected capacity limits at major airports have triggered research toward reducing aircraft separation of approaching and departing aircraft [1-6]. Up-to-date fixed aircraft separation distances are prescribed to avoid wakevortex encounters. The aircraft wake-vortex system consists of two counter-rotating vortices for which the strength is dependent on the weight, span, and speed of the aircraft. Wake vortices descend due to their mutually induced downward velocity. The International Civil Aviation Organization (ICAO) has proposed three aircraft weight categories, with respective separation distances depending on the leading and following aircraft. Those separations have proved to be safe and are often considered as overconservative [ㅇ, 7]. It appears that there is potential for a safe reduction of separation distances; however, this requires that the transport and decay of wake vortices out of the glide-path corridor has to be predicted.

To date, no operational wake prediction and monitoring system exists. The most advanced system has been developed by the German air navigation provider DFS at Frankfurt airport. DFS has developed and installed a ground-based system for their closely spaced parallelrunway system (runway 25L/R) using an anemometer array to track wake vortices near the runway threshold [1]. The wake-vortex warning system in Frankfurt focuses on the lateral transport of wake vortices and employs a statistical crosswind prediction algorithm [8], together with a simple wake-vortex transport and decay model. Recently, DFS deployed a wind and temperature profiler to extend the existing ground-based prediction system to the glide path [9]. With this extension, safe crosswind regimes can be predicted that clear the glide slope from wake vortices. The glide-path extension does not consider vertical wake transport and wake decay. With the given crosswind conditions at Frankfurt airport, only a very marginal capacity gain could be achieved [9]].

Local meteorological measurements at high spatial and temporal resolution are an important element of a wake prediction and observation system at an airport [10]. Ideally, the vertical resolution should be on the order of $50 \mathrm{~m}$, with the temporal resolution on the order of 2-10 min. Full coverage of the whole flight corridor in a terminal area using existing sensor technology is challenging, due to costs and sensor limitations in all weather conditions. Within DLR's national project "Wirbelschleppe," a different approach is pursued by using high-resolution numerical weather forecast to provide consistent data for wake prediction at high temporal and spatial resolutions in the terminal area. For multiple purposes, a one-year meteorological database for the Frankfurt terminal area has been 
generated recently using the model system of nowcasting wakevortex impact variables (NOWVIV). This one-year data set comprises typical weather conditions and includes already-typical features of a long-term surface wind climatology. It enables us to test new operational concepts with realistic meteorological input and to estimate the potential for aircraft separation reduction. It may also be used within risk assessments for prototype wake-vortex advisory systems $[6,11,12]$.

A subset of the one-year database has been analyzed in detail for a period of 40 days, during which a dedicated wake measurement campaign was carried out at Frankfurt airport in fall 2004 [13]. In total, 231 wake-vortex pairs generated by heavy aircraft in ground proximity [in ground effect (IGE)] were tracked and characterized by lidar. In addition, 233 wake vortices were measured out of ground effect (OGE) approximately $5 \mathrm{~km}$ away from the runway threshold. During this measurement campaign, a sonic detection and ranging (SODAR) system, together with a radio acoustic sounding system (RASS) and a lidar, provided profile measurements of meteorological variables. These data were used to analyze the quality of the predicted profiles of wind, temperature, and turbulence [13].

We couple weather prediction and observation with the probabilistic two-phase wake-vortex transport and decay model (P2P) [14-17] and the safety-area prediction model (SHAPE) $[18,19] . \bar{W}$ investigate the predictive skill of $\mathrm{P} 2 \mathrm{P}$ for preselected confidence levels of vortex position and strength using meteorological input from forecast and observations. The skill is assessed by comparing the predictions against lidar-measured wake-vortex position and strength. Initially, the overall skill of the wake-vortex forecast is analyzed. We then analyze the skill of the models with respect to observed and predicted clearance of an approach corridor from wave vortices. In particular, we focus on the frequency of nonconservative predictions, which refer to a situation in which the safety area of a wake vortex is predicted to be outside a predefined approach corridor, although observations still indicate that the safety area of a wake vortex still intersects with the approach corridor. Nonconservative predictions refer to a potential encounter risk for a following aircraft, which has to be avoided.

The use of a numerical weather forecast with local weather observation in a wake prediction and observation system is not a new idea and concept designs have been around for years $[3,6]$. The system must result in a capacity gain that has to be achieve at a very high level of safety to meet the safety requirements defined by authorities. To cover the full variability of wake evolution in the atmosphere, probabilistic modeling approaches are necessary. So far, no analysis has been published in which the combination of weather prediction, weather observation, probabilistic wake predictor, and wake safety-area prediction has been analyzed quantitatively. In this paper, we carry out such an analysis considering a single-runway approach. Previous work focused on the analysis of elements of a wake prediction system without considering the full chain of elements needed to end up with a forecast of corridor clearance time at a preselected probability level. The performance of wake predictors driven by meteorological data measured locally by a suite of sensors was considered [3] in which the variability of weather parameters was considered. This may be viewed as a probabilistic component of the wake prediction system. Principle aspects of wake prediction performance using dedicated numerical weather prediction have been discussed only in a qualitative manner [3].

In the following, we briefly introduce the components of the wake prediction system: namely, the weather forecast system NOWVIV, the probabilistic wake-vortex transport and decay model P2P, and the safety-area prediction mode SHAPE. We then introduce the wake and weather measurement campaign, which is used to analyze the skill and performance of the wake prediction system for wakes evolving in and out of ground effects.

\section{Model System NOWVIV}

A hierarchy of weather forecast models is combined within the model system NOWVIV. The core of NOWVIV is the mesoscale model MM5 [20,21], in which a Yamada-Mellor 2.5-level turbulence closure scheme is employed and turbulent kinetic energy is computed as a prognostic variable. Two nested domains with sizes of approximately $250 \times 250$ and $90 \times 90 \mathrm{~km}^{2}$ centered on Frankfurt airport with grid distances of 6.3 and $2.1 \mathrm{~km}$, respectively, are used. The model employs 60 vertical levels such that in the altitude range of interest $(z<1100 \mathrm{~m}$ above ground), 26 levels yield a vertical resolution varying between 8 and $50 \mathrm{~m}$. Initial and boundary data are taken from the analysis run of the mesoscale model Lokal Modell (LM) [22] of Deutscher Wetterdienst (DWD, German Weather Service).

Detailed terrain and land use information is provided to NOWVIV, which is initialized every $12 \mathrm{~h}$, at 0 and 12 Coordinated Universal Time (UTC). Locally measured data have not been assimilated for this test. Output variables are vertical profiles of horizontal and vertical winds $u, v$, and $w$; virtual potential temperature $\theta_{v}$; and turbulent kinetic energy $e$.

\section{Measurement Campaign}

A dedicated wake measurement campaign was carried out at Frankfurt airport in the fall of $2004[13,17]$. A suite of sensors and the forecast model NOWVIV provided the meteorological data for the evaluation of the wake prediction system. A detailed data comparison of meteorological measurements and predictions for a time period of 40 days can be found in [13]. The measurements were taken at a site close to the runway threshold of $25 \mathrm{~L} / \mathrm{R}$ (Fig. 1). This time period includes a whole range of synoptic conditions, from latesummer high-pressure situations to frontal passages with strong winds and precipitation [13].

The SODAR/RASS measurements provide 10-min averaged profiles of all three wind components, standard deviations of vertical velocity, and virtual temperature. The vertical resolution of the profiles is $20 \mathrm{~m}$, and the first measurement level is $40 \mathrm{~m}$ (which represents an average between 30 and $50 \mathrm{~m}$ ). The vertical availability of meteorological parameters varies with time, depending on environmental (aircraft noise) and meteorological conditions. With the chosen settings, the vertical availability of SODAR/RASS data is typically on the order of 200-300 m. The accuracy of the wind-speed measurement depends on the wind-speed magnitude. Up to $5 \mathrm{~m} / \mathrm{s}$, the accuracy is within $\pm 0.5 \mathrm{~m} / \mathrm{s}$, and from $5-35 \mathrm{~m} / \mathrm{s}$, the accuracy is within $\pm 10 \%$. For wind direction, the accuracy is $\pm 5 \mathrm{deg}$. The standard deviation of vertical wind velocity can be estimated within $\pm 0.15 \mathrm{~m} / \mathrm{s}$. In addition, an ultrasonic anemometer at $z=10 \mathrm{~m}$ was operated close to the SODAR/RASS measurements. The sonic

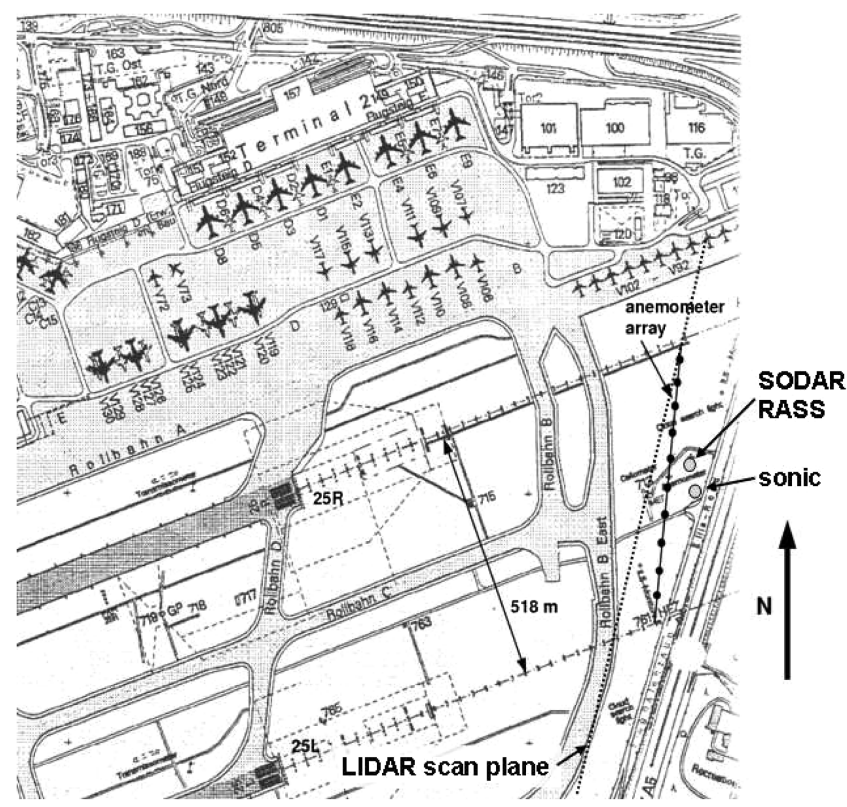

Fig. 1 Layout of sensor location near the thresholds of runway 25L/R. Also shown is the DFS anemometer array and the DLR lidar scan plane. 


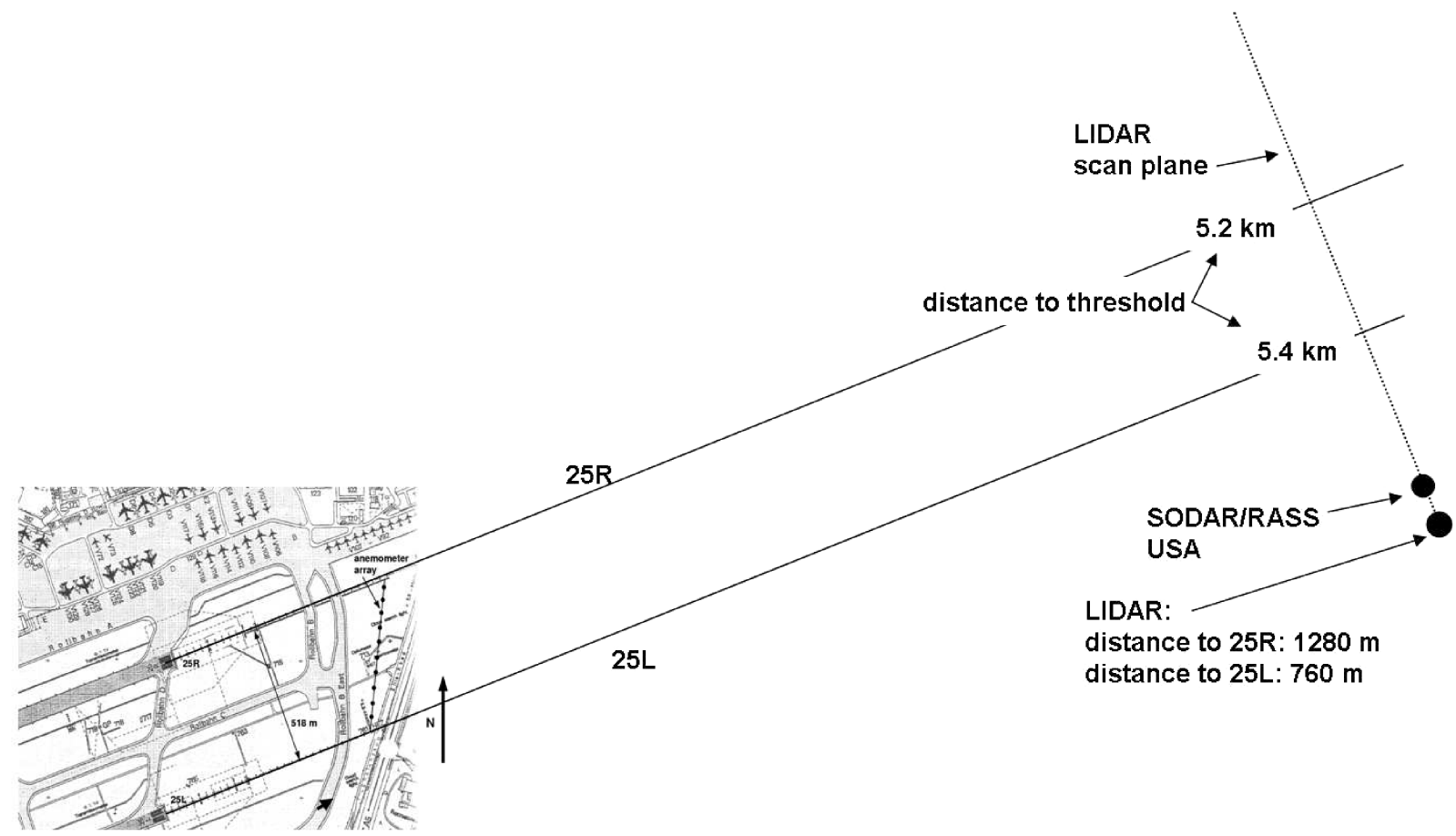

Fig. 2 Measurement layout of the OGE measurement. WVs are generated in the lidar scan plane at a height of $\approx 270 \mathrm{~m}$.

anemometer provides measurements of all three wind components and temperature at a sampling rate of $17 \mathrm{~Hz}$.

In total, 231 wake-vortex pairs generated by A340 and B747 aircraft during final approach at a nominal height of approximately $60 \mathrm{~m}$ above the ground were traced with a $2-\mu \mathrm{m}$ pulsed-lidar system. The lidar scanned the measurement plane at an angle of 123 deg to flight direction, employing elevation sectors of 0 to $15 \mathrm{deg}$. For the evaluation of wake-vortex properties, an interactive four-stage data processing algorithm was applied $[23,24]$. From estimated profiles of vortex tangential velocities, vortex positions and circulations were derived.

In addition, 233 wake vortices were measured out of ground effect approximately $5.3 \mathrm{~km}$ from the threshold at which the aircraft are approximately $270 \mathrm{~m}$ above ground level. The measurement layout is shown in Fig. 2.

\section{Probabilistic Wake-Vortex Prediction Model}

The real-time probabilistic two-phase aircraft wake-vortex model P2P considers all of the first-order wave vortices impact parameters: namely, the aircraft configuration, wind, turbulence, temperature stratification, windshear, and proximity of the ground [14-17]. The model is formulated in normalized form, in which the characteristic scales are based on initial vortex separation $b_{0}$ and circulation $\Gamma_{0}$, leading to the time scale $t_{0}=2 \pi b_{0}^{2} / \Gamma_{0}$.

For the prediction of circulation, the concept of two-phase circulation decay is pursued, in which a turbulent-diffusion phase is followed by a rapid-decay phase (see Fig. 3). This behavior has been adopted from large eddy simulation results of wake-vortex evolution in turbulent and stably stratified environments and, in the meantime, has also been confirmed by lidar observations [16]. Aloft, the onset time of rapid decay depends on ambient turbulence and stratification. In ground proximity, however, the decay rate is mainly controlled by the interaction of the wake vortex with secondary vortices that detach from the boundary layer at the ground. Consequently, in ground effect, the decay rate depends only weakly on ambient meteorological conditions. On the other hand, the impact of crosswind on vortex rebound characteristics is very strong [17]. Crosswind attenuates (intensifies) the formation of the luff (lee) secondary vortex, which causes pronounced asymmetric rebound behavior (see Fig. 3).
To consider spatiotemporal variations of vortex position and strength, which are caused primarily by turbulent transport and deformation processes, the probabilistic model predicts wave-vortex behavior within defined confidence intervals (see Fig. 3). For this purpose, decay parameters are varied in consecutive model runs, and
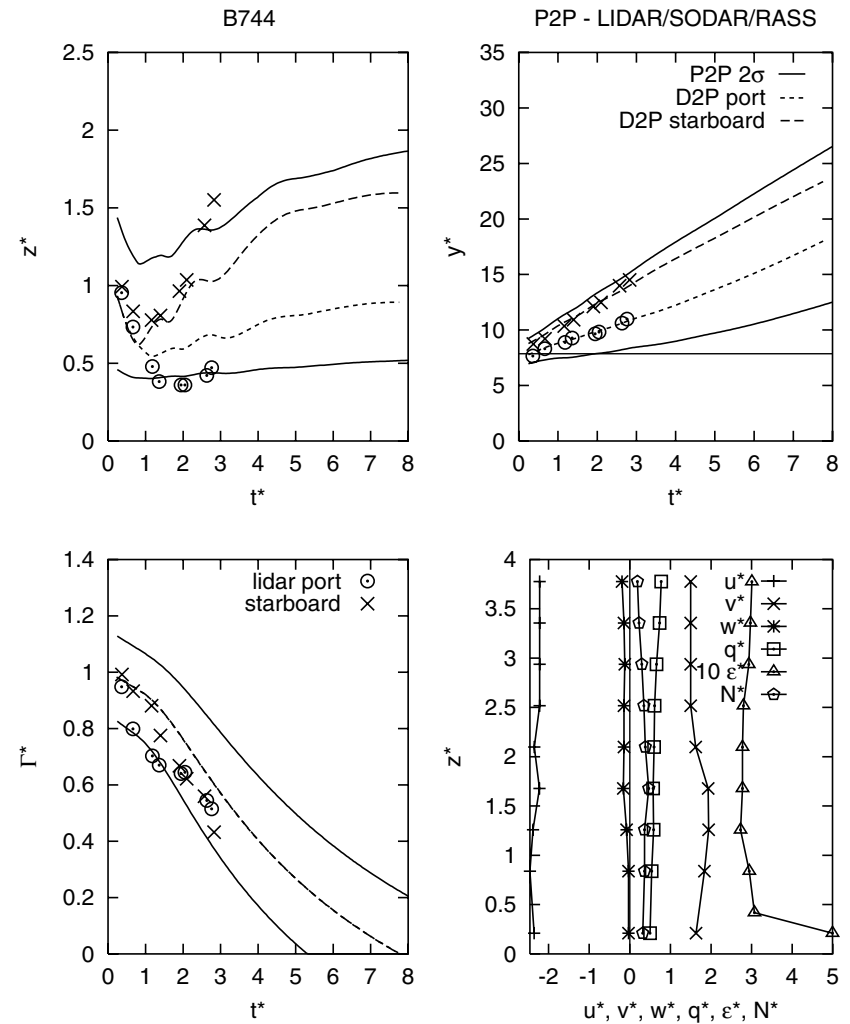

Fig. 3 P2P predictions with SODAR/RASS input. Measured (symbols) and predicted (lines) evolution of normalized vertical and lateral positions $z^{*}$ and $y^{*}$ and circulation $\Gamma^{*}$ in ground proximity. Dashed lines denote deterministic behavior, and solid lines denote envelopes for probabilities of $95.4 \%$. The bottom right plot shows vertical profiles of normalized environmental data. 

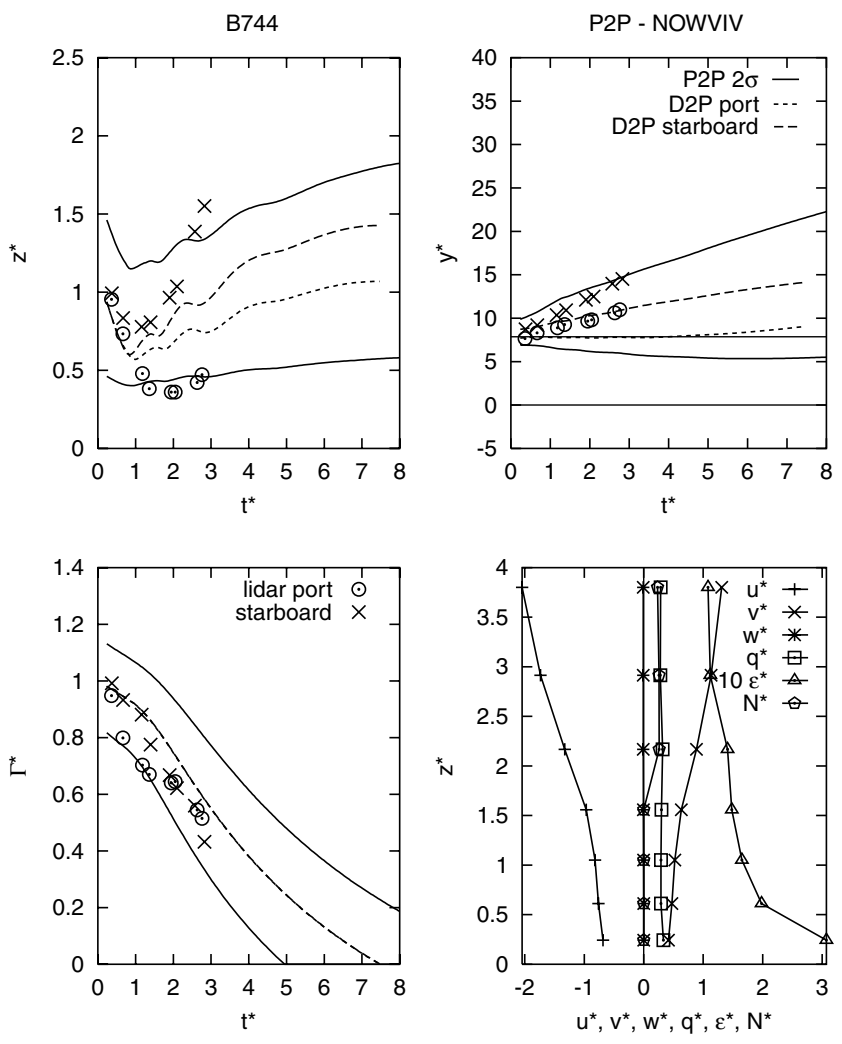

Fig. 4 P2P predictions with NOWVIV input (see also the same overflight in Fig. 3).

various static and dynamic uncertainty allowances are added that consider the increased scatter of wake-vortex position and strength in turbulent environments and their modified trajectories caused by tilting and rebound in windshear situations. The obtained probabilistic envelopes can be adjusted to represent preselected degrees of probability. The respective envelopes are estimated based on a training procedure [16] that relates the predicted envelopes to field measurement data. To achieve consistency between wind and wake prediction skill, separate fits are used for wake predictions driven by predicted and by measured environmental parameters. That is, the predicted probabilistic envelopes fully consider the uncertainties of both meteorological input parameters and wakevortex model parameterizations. A deterministic model version termed D2P provides mean wake-vortex evolutions employing intermediate decay parameters.

Figure 3 displays an extreme example in which the asymmetric rebound is very pronounced, such that evolutions of vertical vortex position exceed the $2 \sigma$ envelopes $(95.4 \%)$. Figure 4 shows the same overflight based on a relatively poor NOWVIV crosswind prediction. Note that the spread of the probabilistic envelopes for lateral position $y^{*}$ is increased compared with the SODAR-driven predictions, due to crosswind prediction uncertainties.

\section{Simplified Hazard-Area Prediction Model}

P2P only predicts the probability of wake strength and position. The dimension of the area around the wake-vortex position that has to be avoided by a follower aircraft for safe and undisturbed flight must be predicted by another model. Here, we employ the simplified hazard-area prediction method SHAPE, which assumes a ratio of 0.2 between the rolling moment exerted by the wake-vortex pair and the available aircraft roll-control capability to compensate the induced rolling moment [19]. Below this threshold, a wake encounter can be controlled by an aircraft, which has been shown in full flight simulator investigations complemented by real flight tests using DLR's fly-by-wire in-flight simulator ATTAS (Advanced Technologies Transport Aircraft System). In a parameterized version of SHAPE, we determine the required lateral and vertical

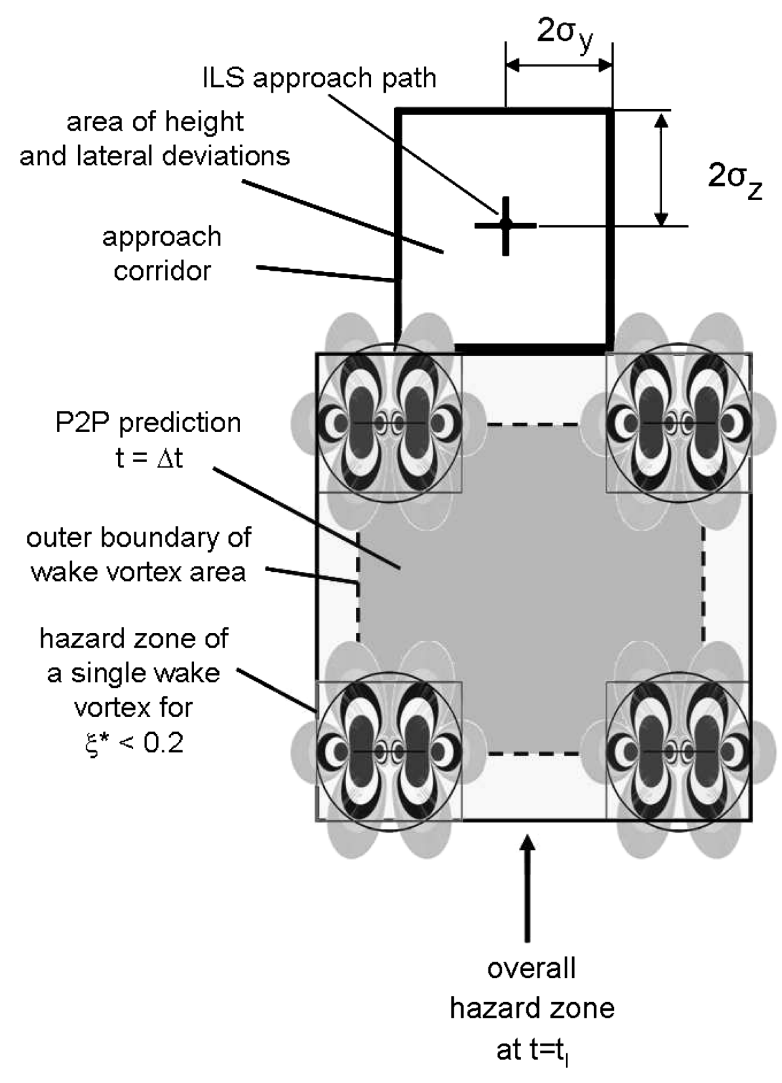

(penetration prohibited)

Fig. 5 Schematic graph showing the approach corridor, the wakevortex area, and the respective safety zones. The resulting overall safety zone is shown at time $t_{l}$, when there is no longer an overlap with the approach corridor. The dimensions of the approach corridor consider the uncertainty of aircraft to follow the corridor.

distances from the vortex center as functions of the predicted circulation. These can be computed for a heavy-heavy $(\mathrm{HH})$ aircraft pairing or a heavy-medium (HM) pairing in which the safety allowances are evaluated using characteristic aircraft parameter combinations such as span, weight, and speed in the heavy- and medium-weight categories. As an example, for a given circulation of $\Gamma=300 \mathrm{~m}^{2} \mathrm{~s}^{-1}$, a roll-control ratio of 0.2 is found at $33 \mathrm{~m}(51 \mathrm{~m})$ in lateral and $30 \mathrm{~m}(58 \mathrm{~m})$ in vertical distances from the vortex center for a heavy (medium) follower. In Fig. 5 , the respective four safety zones of wake vortices located around the corners of the predicted boundary of the wake-vortex area at a time $t_{l}$ are schematically shown. The upper circulation limit of the $\mathrm{P} 2 \mathrm{P}$ prediction is taken to compute the SHAPE safety allowance. Using the SHAPE safety allowance, we then can determine an overall safety zone that has to move out of the static approach corridor. The time for which this occurs determines a safe separation time for a following aircraft and thus allows for an assessment of a potential capacity gain compared with ICAO separations.

\section{Skill of Wake-Vortex Predictions in Ground Effect}

Especially in ground proximity, crosswind-driven wake-vortex drift constitutes the primary mechanism to clear the approach corridor from wake vortices. For an operational wake-vortex advisory system, the performance of crosswind predictions is decisive. We therefore compare the model forecast not only against observations, but also against a crosswind prediction based on a persistence model. We assume that the currently measured crosswind is valid up to a given lead time in the future. For analysis purposes, we consider a forecast lead time of up to $6 \mathrm{~h}$ to identify the lead time for which we can expect a superior model forecast. The crosswind thresholds considered are 2 and $3 \mathrm{~m} / \mathrm{s}$, respectively. The 


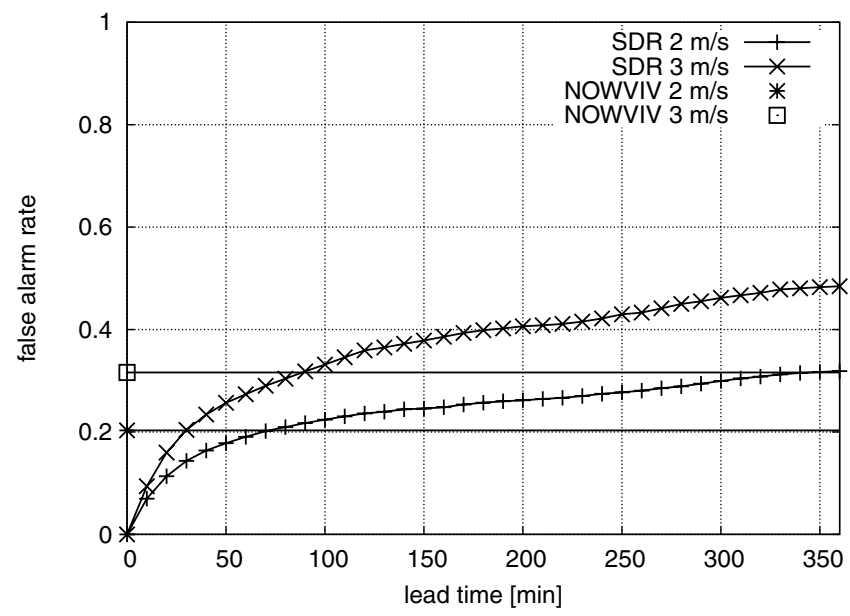

Fig. 6 The false-alarm rate regarding exceedance of crosswind thresholds of 2 and $3 \mathrm{~m} / \mathrm{s}$ for a SODAR persistence forecast and the NOWVIV forecast based on 40 measurement days.

requirement is that the exceedance of a given crosswind threshold has to be predicted correctly over the whole crosswind profile. From a contingency table, we compute the false-alarm rate (FAR) [25]. A false alarm refers to a situation in which the predicted crosswind is above the threshold, whereas the measured crosswind is below the specified crosswind threshold. The result is shown in Fig. 6. The FAR of the NOWVIV forecast is plotted on the $y$ axis as a single point that is extended over the whole lead-time range of the SODAR crosswind persistence forecast, for an easier analysis of the results. Overall, the FAR increases with increasing crosswind threshold. The FAR of NOWVIV is $0.2(0.32)$ for a crosswind threshold of $2 \mathrm{~m} / \mathrm{s}$ $(3 \mathrm{~m} / \mathrm{s})$. We find that the skill of the persistence forecast up to a lead time of $60 \mathrm{~min}(80 \mathrm{~min})$ is superior compared with the NOWVIV performance. Beyond this lead time, NOWVIV shows a better forecast performance.

It has to be noted that we use the direct model output without any further optimization by using, for example, local observations. An approach referred to as model output statistics (MOS) is commonly employed by weather services to correct direct model output and to assign confidence levels to forecasted parameters. Certainly, there is potential to reduce the NOWVIV FAR by introducing safety allowances.

To investigate the impact of meteorological data on wake-vortex behavior as it is done within a wake-vortex advisory system, we couple the NOWVIV forecast and SODAR measurements with the P2P wake-vortex model.

We first analyze the overall forecast performance of P2P using NOWVIV and the simple crosswind persistence forecast based on SODAR data as input. We employ a scoring procedure that considers, in total, 231 IGE and 233 OGE lidar-measured wake vortices serving as a reference for the evaluation of the $\mathrm{P} 2 \mathrm{P}$ results. The scoring procedure evaluates the root-mean-square deviations of measurement and prediction of the quantities $y^{*}, z^{*}$, and $\Gamma^{*}$ for each overflight. From the distribution of rms values resulting from the 231 cases, the median and the 90th percentile are used to characterize the performance of the models. This scoring approach is based on a deterministic version of P2P (D2P), whereas an operational system would employ the fully probabilistic version of P2P.

The scoring results shown in Fig. 7 indicate that the root-meansquare deviations between D2P predictions and observations of the lateral wake-vortex position using NOWVIV input initially are approximately a factor of 2 larger than the deviation using SODAR input. For a typical large aircraft with an initial vortex spacing of $b_{0}=45 \mathrm{~m}$, the rms error using SODAR input is on the order of $20 \mathrm{~m}$ for the lateral vortex position and only $7 \mathrm{~m}$ for the vertical vortex position. For completeness, we also show the 90th percentile of the rms error distribution, which displays characteristics similar to the median (Fig. 7, lower panel). The 90th percentile for the SODARdriven prediction is near $y^{*}=1$ and 2.1 for the NOWVIV case.
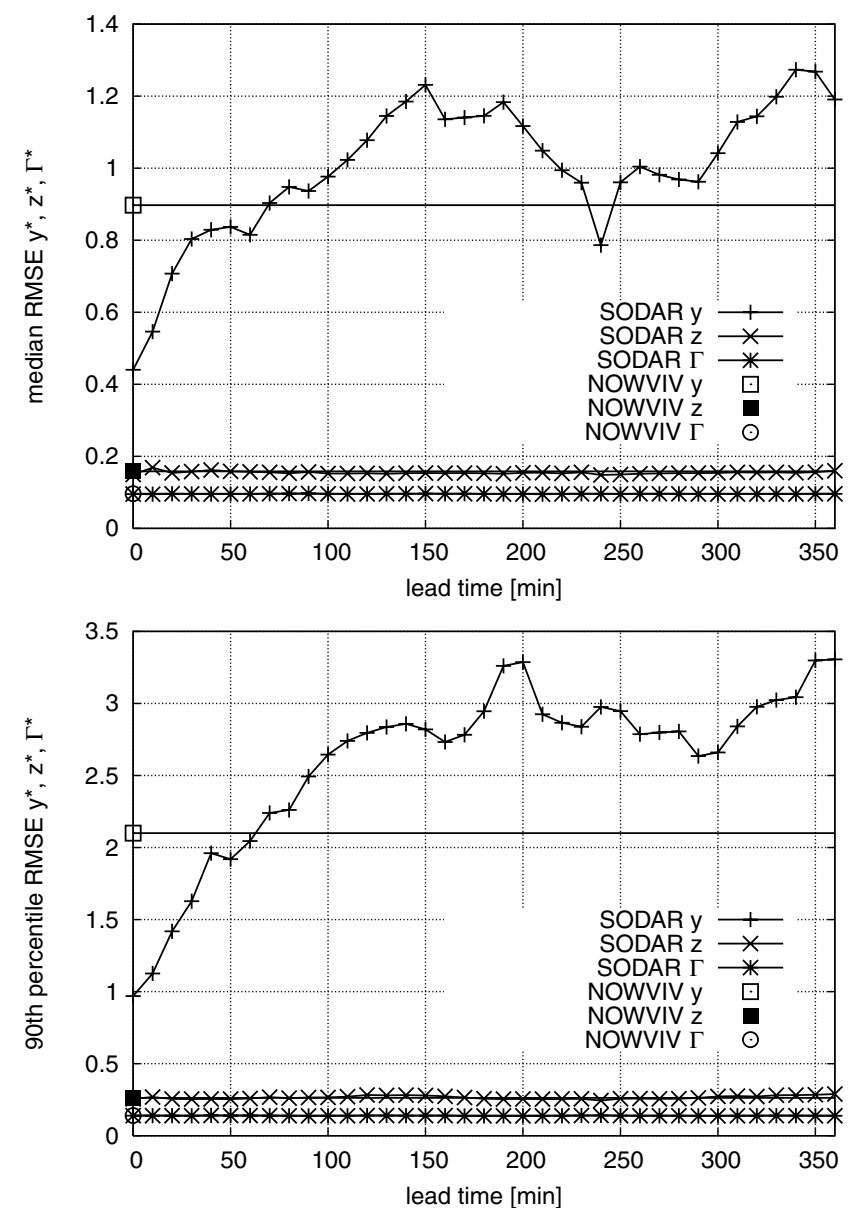

Fig. 7 Median (upper panel) and 90th percentile (lower panel) of the normalized rms error distribution of the predicted lateral and vertical wake-vortex positions and circulation (here, the IGE data).

As one could expect, the rms error of lateral position increases with increasing lead time using the SODAR-based persistence model. The crossover time is found for a lead time of $t_{\text {lead }} \approx 60 \mathrm{~min}$, which approximately corresponds to the crossover time we found for a crosswind threshold of $2 \mathrm{~m} / \mathrm{s}$ with respect to FAR (see Fig. 6).

The rms errors of the vertical position and circulation are nearly identical for both numerical prediction and persistence forecast, which suggests that these quantities are independent of meteorological conditions, which is also reflected by the fact that the errors are essentially constant with increasing lead time. Note that crosswind leads to pronounced asymmetric rebound behavior (Figs. 3 and 4). However, this is mainly controlled by the crosswind sign, which is obviously a well-predicted quantity, and only slightly controlled by crosswind magnitude [17].

Previous analysis concentrated on the skill of the deterministic version of $\mathrm{P} 2 \mathrm{P}$ compared with lidar-measured wake vortices. We now focus on the performance of the fully probabilistic version of the model to predict the time when a wake vortex leaves a approach corridor, $t_{l}$. The requirement is that $t_{l, \text { observed }} \leq t_{l \text {,predicted }}$. In the lateral direction, the approach corridor has a width of $\pm 28 \mathrm{~m}$ from the runway centerline, which corresponds to a $2 \sigma$ probability of glidepath adherence statistics of the FLIP (Flight Performance Using Frankfurt ILS) study. FLIP quantifies lateral and vertical aircraft deviations from the instrument landing system (ILS) of Frankfurt airport [26]. In the analysis, we consider only WVs that were observed by the lidar at least for $60 \mathrm{~s}$, and so seven wake measurements had to be removed from the sample.

Figure 8 shows the skill obtained using P2P predictions with a $2 \sigma$ confidence level $(95.4 \%)$. There, both SODAR- and NOWVIVdriven $\mathrm{P} 2 \mathrm{P}$ versions demonstrate nearly equal skill at $t_{\text {lead }}=0$. Both forecasts show a close-to-perfect performance. The skill of the 


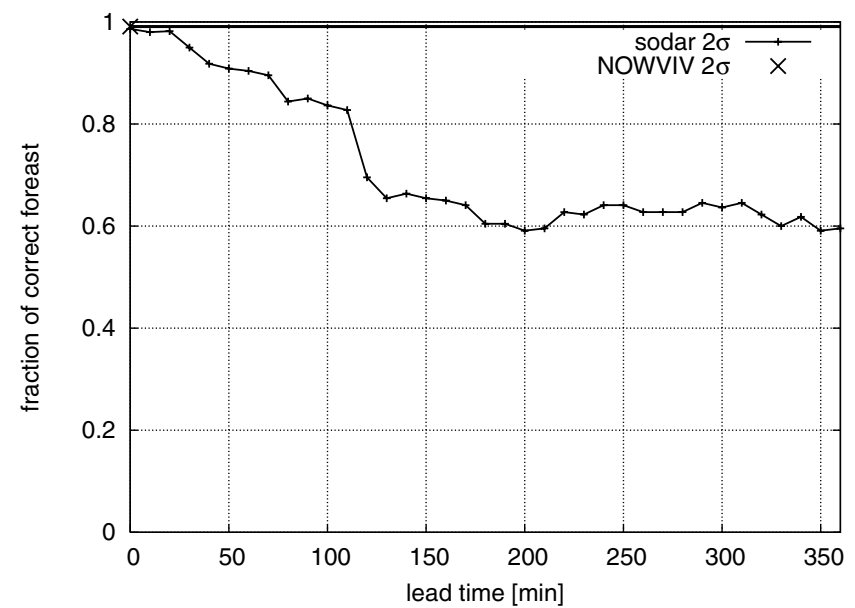

Fig. 8 The fraction of model forecasts that correctly predicts the time to leave the corridor, $t_{l}$. The requirement is that $t_{l, \text { observed }} \leq t_{l, \text { predicted }}$. Shown are P2P results based on $2 \sigma$ confidence levels using SODAR and NOWVIV input.

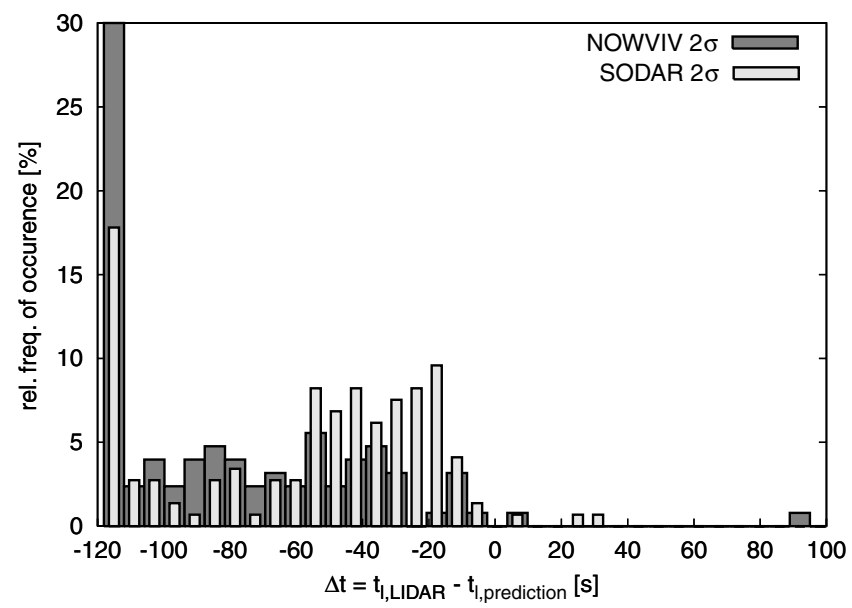

Fig. 9 Histograms of the difference between the observed and predicted time to leave the corridor $\left(t_{l, \text { lidar }}-t_{l, \text { prediction }}\right)$ for the probabilistic predictions.

NOWVIV-P2P predictions can be attributed to the P2P safety allowances, which were adapted to the NOWVIV predictions and thus led to good results even for large uncertainties in predicted meteorological input. For a lead time of $30 \mathrm{~min}$, the persistence approach (SODAR-P2P) still correctly predicts the residence time in $94 \%$ of the cases. The quality of the $\mathrm{P} 2 \mathrm{P}$ forecast driven by SODAR starts to degrade substantially for lead times beyond $\approx 60 \mathrm{~min}$.

A small number of SODAR-P2P and NOWVIV-P2P predictions appeared to be nonconservative (a fraction of the correct forecast, less than one). An impression of the associated errors can be obtained by evaluating the corresponding histograms of the difference between the observed and predicted times to leave the corridor: $\Delta t_{l}=t_{l, \text { lidar }}-t_{l, \text { prediction }}($ see Fig. 9).

The histograms for the $\mathrm{P} 2 \mathrm{P}$ runs with the $2 \sigma$ confidence level show three forecasts for SODAR-P2P and two forecasts for NOWVIV$\mathrm{P} 2 \mathrm{P}$ with $\Delta t_{l}>0 \mathrm{~s}$, which indicates a high level of safety of the predictions (greater than 95.4\%). P2P output is trained to achieve a $95.4 \%$ confidence level over the observed life span of a wake vortex. The histogram shows the necessity to use a $2 \sigma$ confidence level to keep the number of false predictions below $4.6 \%$.

\section{Skill of Wake-Vortex Predictions Out of Ground Effect}

We now investigate the skill of SODAR-P2P and NOWVIV-P2P for wake predictions OGE. Case realizations of wake-vortex predictions versus measurements OGE can be found in [14-16]. The
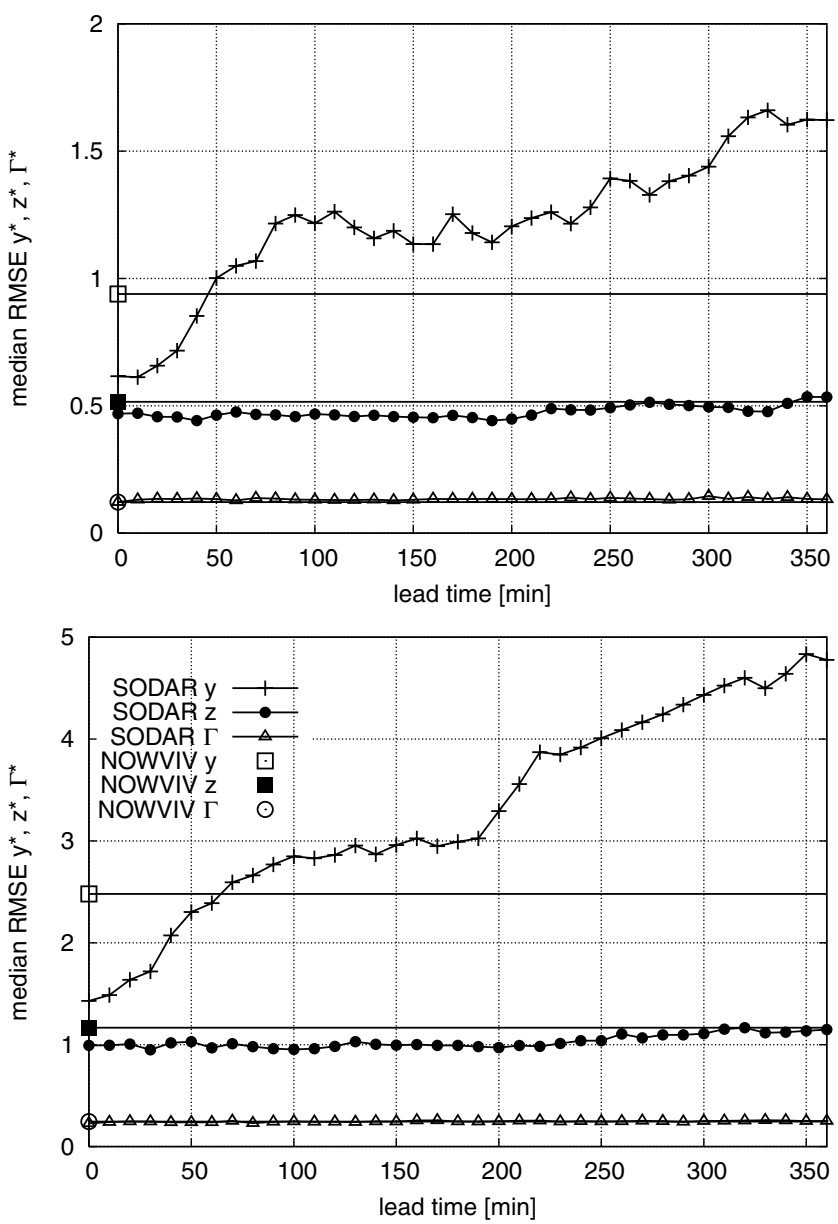

Fig. 10 Median (upper panel) and 90th percentile (lower panel) of the normalized rms error distribution of the predicted lateral and vertical wake-vortex positions and circulation (here, the OGE data).

scoring results of D2P for the OGE data are shown in Fig. 10. Similar to the IGE data, D2P predictions of the lateral wake-vortex position using NOWVIV input initially are approximately a factor of 2 larger than the predictions using SODAR input. Compared with Fig. 7, we notice that the initial scoring of the lateral and vertical vortex positions of the IGE cases at $t_{l}=0 \mathrm{~s}$ is superior to the OGE cases. Essentially, no differences are found for the $\Gamma$ scoring. Lower D2P errors IGE relate to lower uncertainty of the meteorological input data due to the measurement layout and the restriction of vertical transport by the land surface. The crossover time for superior SODAR-P2P skill is found for a lead time of approximately $50 \mathrm{~min}$.

The vertical error in position of the persistence forecast is smaller than the NOWVIV-P2P error and shows a 12\% increase with lead time. The magnitude of the circulation error is nearly identical for inputs using the persistence forecast and the numerical weather prediction. The circulation error of the persistence approach shows a $10 \%$ increase. The moderate increase of errors in decay due to stratification and turbulence is remarkable because we consider a lead time up to $6 \mathrm{~h}$.

Similar to the IGE analysis, we investigate the skill of P2P to predict the time to leave the flight corridor $t_{l}$. We initially consider the skill of the wake predictions assuming only lateral transport to clear the approach corridor before we also account for vertical transport.

Based on the FLIP data, we can expect $\sigma_{y}=15 \mathrm{~m}$ for the lateral standard deviation from the ILS and $\sigma_{z}=10 \mathrm{~m}$ for the vertical deviation. The corridor is assumed to have a rectangular shape with dimensions of $2 \sigma_{y} \cdot 2 \sigma_{z}=60 \cdot 40 \mathrm{~m}^{2}$.

The mean vertical and lateral positions of the aircraft when it passes the lidar scan plane is computed from the wake trajectories, the known overflight time, and the measured crosswind. For our 


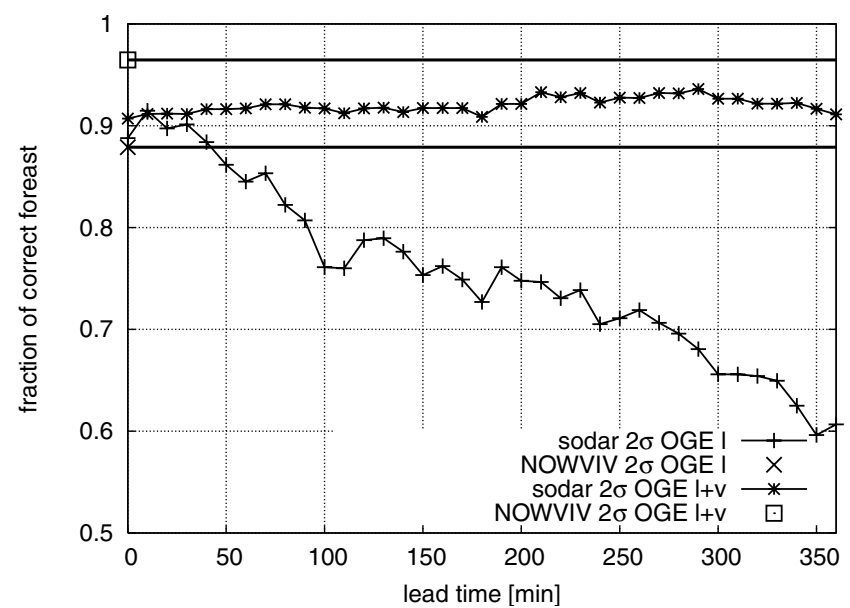

Fig. 11 The fraction of model forecasts OGE that correctly predict the time to leave the corridor, $t_{l}$. Lateral transport out of the approach corridor is denoted by subscript $l$, and lateral and vertical transport is denoted by $l+v$. The requirement is that $t_{l, \text { observed }} \leq t_{l, \text { predicted }}$. Shown are P2P results using $2 \sigma$ confidence levels using SODAR and NOWVIV input.

small sample of aircraft, we find a deviation around the mean aircraft position of $\sigma_{z}=11 \mathrm{~m}$ and $\sigma_{y}=45 \mathrm{~m}$. The deviation in the vertical direction agrees well with the FLIP value, whereas the lateral deviation is significantly larger. $\mathrm{P} 2 \mathrm{P}$ predictions are initialized for each individual wake vortex at the respective reconstructed glidepath position.

The $\mathrm{P} 2 \mathrm{P}$ results with $2 \sigma$ safety allowances for the OGE data are shown in Fig. 11. In this figure, graphs denoted with $l$ refer to approach corridor clearance due to lateral wake transport only, whereas $l+v$ also accounts for the vertical transport of the wake out of the approach corridor.

If we consider lateral transport only, SODAR-P2P initially predicts the correct $t_{l}$ approximately $90 \%$ of the time, compared with $88 \%$ for NOWVIV-P2P. Better skill of SODAR-P2P is found up to a lead time of $40 \mathrm{~min}$ (Fig. 11). If we also consider the vertical transport out of the corridor, SODAR-P2P correctly predicts the clearance time in $92 \%$ of the cases, whereas NOWVIV-P2P predicts correct clearance in $97 \%$ of the cases. A remarkable aspect is that the skill of SODAR-P2P is more or less independent of lead time if we consider the vertical transport. This suggests that the wake evolution in the early stage appears independent of atmospheric conditions. Before stratification or turbulence may cause a deceleration or even rebound, the wake vortex has moved out of the approach corridor by mutual induction. This independence may open ground for very simplified wake transport models within wake avoidance systems, because it appears to be unnecessary to predict the wake evolution of approaching aircraft individually. Surprisingly, NOWVIV-P2P shows a better skill compared with SODAR-P2P, which may be explained by larger NOWVIV-P2P safety allowances to account for forecast uncertainties. This will be considered later, when we analyze the potential capacity gain or loss.

Compared with the IGE results, a larger fraction of SODAR-OGE predictions have erroneous corridor clearance times. On the other hand, the difference between prediction and observation is smaller than $5 \mathrm{~s}$ (Fig. 12). These cases relate to low $t_{l}$ values for both observation and prediction. On average, the observed wakes leave the corridor within $16 \mathrm{~s}$, compared with $14 \mathrm{~s}$ predicted by SODAR$\mathrm{P} 2 \mathrm{P}$, suggesting that this bias is not of relevance for operational applications in which aircraft are at least separated by radar separation $(\approx 60 \mathrm{~s})$. Therefore, the skill of SODAR-P2P can be considered as safe.

\section{Coupling of P2P with SHAPE and the Potential Capacity Gain}

In this section, P2P and SHAPE are coupled to determine not only the predicted wake-vortex residence area, but also the overall safety

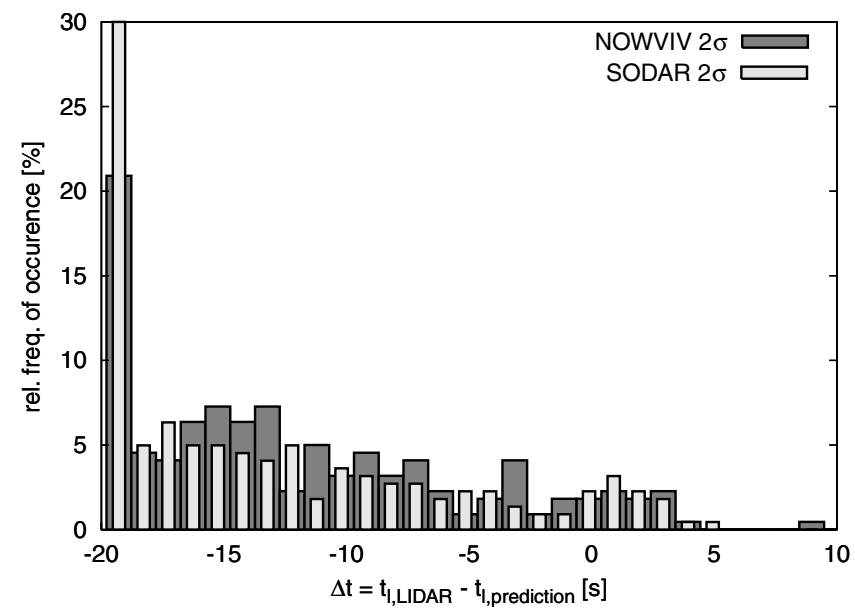

Fig. 12 Histograms of the difference between the observed and predicted time to leave the corridor $\left(t_{l, \text { lidar }}-t_{l, \text { prediction }}\right)$ for the probabilistic predictions with a $2 \sigma$ safety allowance.

area. This is an important safety aspect, because the predicted circulation strength and position and the effect on a following aircraft is considered explicitly by adding additional safety margins such that any encounter causing rolling moments that exceed $20 \%$ of the rollcontrol capability is avoided.

The capacity potential of the combination weather prediction/ observation, P2P and SHAPE, for a single-runway approach is deduced from the analysis of the cumulative distribution of corridor clearance times. For simplicity, we do not include operational considerations such as a traffic mix. SHAPE safety allowances for a leading heavy aircraft and a following heavy $(\mathrm{HH})$ and medium (HM) aircraft are considered. We use the upper limit of the P2P circulation prediction to compute the lateral and vertical safety allowances, which are added to the predicted $\mathrm{P} 2 \mathrm{P}$ corridor dimensions at which the wake is expected to be at a $2 \sigma$ probability level (see also Fig. 5). SHAPE safety allowances are also added to the lidar data. We compute cumulative distributions of the time to leave the corridor from the P2P forecast using a $2 \sigma$ confidence level and from lidar observations.

The cumulative distribution based on lidar measurements IGE including SHAPE safety allowances indicates that in approximately $90 \%$ of the cases, the corridor is free of potential hazardous wake encounters within $\approx 100 \mathrm{~s}$ (Fig. 13). If we consider a 100-s threshold, the corridor is cleared from potential hazardous wakes using SODAR-P2P forecast for pairings $\mathrm{HH}$ (HM) in $31 \%$ (25\%) of the cases. Similarly, we find $6 \%(4 \%)$ for NOWVIV-P2P forecast for

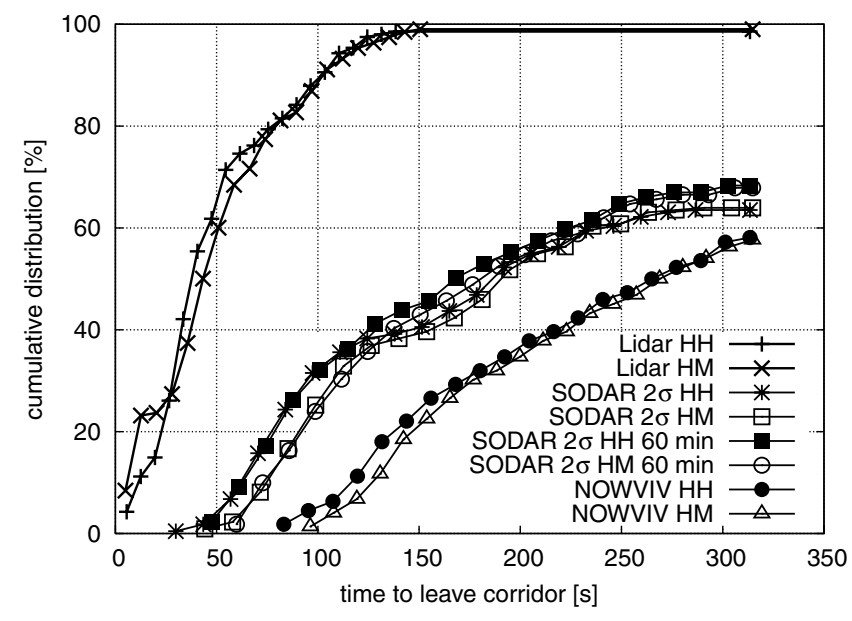

Fig. 13 Cumulative distributions of time to leave the corridor based on IGE observations (lidar) and SODAR-P2P and NOWVIV-P2P with $2 \sigma$ confidence levels including the SHAPE safety allowances for heavy (HH) and medium (HM) follower aircraft. 


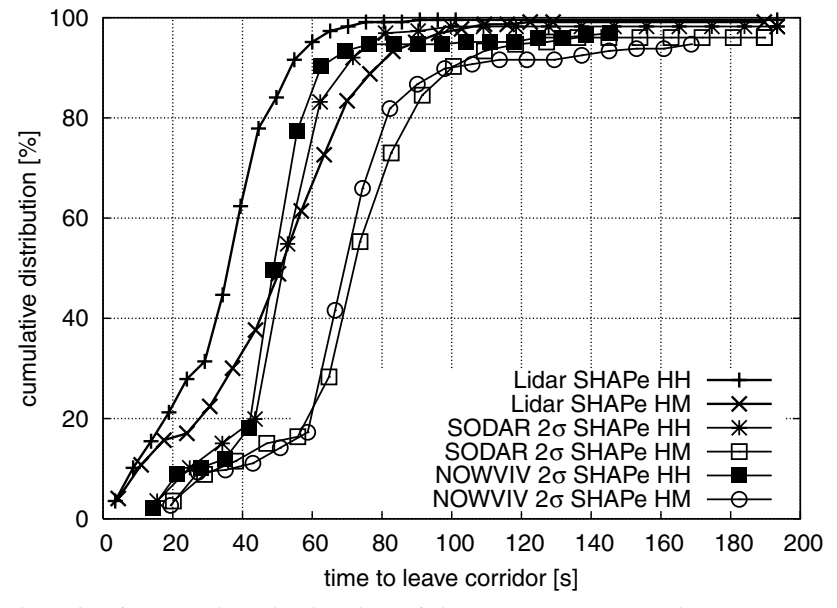

Fig. 14 Cumulative distribution of time to leave the corridor based on OGE observations (lidar) and SODAR-P2P and NOWVIV-P2P with $2 \sigma$ confidence levels including the SHAPE safety allowances for heavy $(\mathrm{HH})$ and medium (HM) follower aircraft.

pairings HH (HM) (Fig. 13). We also note that there is only a weak dependence on lead time for SODAR-P2P (shown are lead times of $t=0$ and $60 \mathrm{~min}$ in Fig. 13), which would facilitate the implementation of such an approach into an operational wake avoidance system. Note that lidar observes vortex behavior only within the observation plane, which means that not all parts of the deformed vortices may have actually left the approach corridor at the times indicated by the lidar measurements plus SHAPE in Fig. 13. This means that the actual potential capacity gain is lower than suggested by the lidar data in Fig. 13. In contrast, the probabilistic predictions including SHAPE specify that all parts of the vortices have left the corridor.

The OGE results show (Fig. 14) that for a medium follower (HM), $95 \%(92 \%)$ of the safety areas have left the approach corridor within $120 \mathrm{~s}$ for SODAR-P2P (NOWVIV-P2P). If we add the SHAPE safety allowance to the measured wake positions, it takes $75 \mathrm{~s}$ to clear the corridor for the heavy follower and $120 \mathrm{~s}$ for the medium follower. This needs to be related to ICAO wake-vortex separations for a heavy-heavy combination ( 4 nm with $t \approx 100 \mathrm{~s}$ ) and heavymedium ( $5 \mathrm{~nm}$ with $t \approx 130 \mathrm{~s}$ ), for which we assume an approach speed of $70 \mathrm{~m} / \mathrm{s}$. Compared with ICAO separations, the results show that a capacity gain appears feasible. Based on the results, it is obvious that the main bottleneck is the timely clearance of the approach corridor close to the surface.

The importance of also considering OGE vertical wake transport is highlighted in Fig. 15. Based on the lidar data, it takes approximately $150 \mathrm{~s}$ before the corridor is cleared from wakes only by lateral

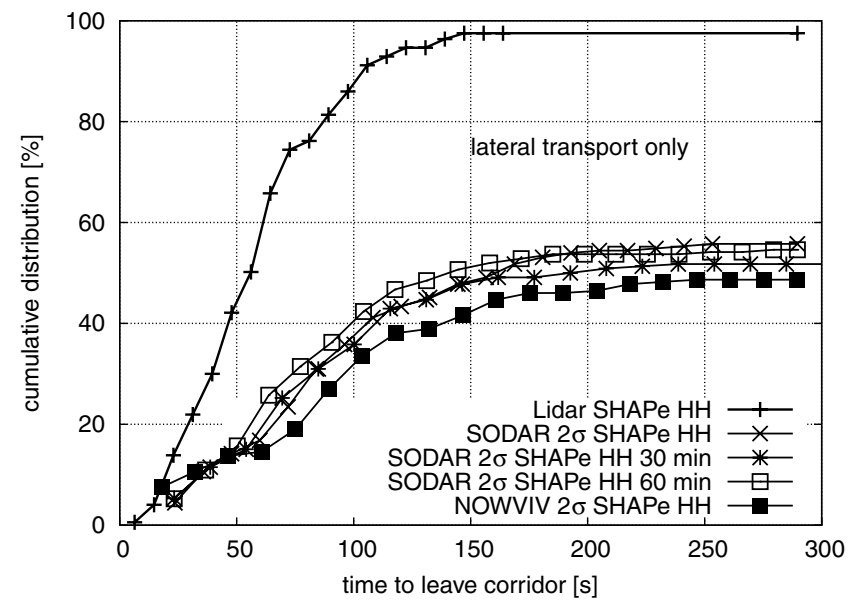

Fig. 15 Cumulative distribution of time to leave the corridor based on OGE observations (lidar) and SODAR-P2P and NOWVIV-P2P with $2 \sigma$ confidence levels allowing only lateral transport to clear the corridor. transport. The results for SODAR-P2P and NOWVIV-P2P suggest that in 40 to $50 \%$ of the cases, there is a $95.4 \%$ probability that wakes remain in the approach corridor, whereas Fig. 14 shows that a large fraction of the wakes have left the corridor within approximately $60 \mathrm{~s}$. This implies that wake avoidance systems give away a significant amount of capacity potential if only lateral wake-vortex transport is considered. Also included in Fig. 15 are results from SODAR-P2P for lead times 30 and $60 \mathrm{~min}$, which indicate the weak dependence on lead time, similar to the IGE results.

Furthermore, the results of NOWVIV-P2P shown in Fig. 14 are promising, because the use of numerical weather prediction allows for a straightforward coverage of the whole glide path, which directly includes the respective mesoscale spatial variability of wind and temperature fields along the glide path [13]

\section{Discussion}

Our analysis confirms previous findings that indicate that the wake behavior in ground proximity represents the bottleneck for wake avoidance systems. The cumulative distributions of the corridor clearance time based on lidar measurements clearly suggest significant capacity potential for single-runway operations, even if we account for the 3-D structure of WVs not visible in a lidar scan plane.

An important aspect of this study is the robustness of vertical wake transport to clear the approach corridor. For our sample taken at Frankfurt airport, it is shown that lateral wake transport out of the approach corridor will not provide significant capacity gain. This is in line with recent findings [9] in which the prediction of lateral wake transport in closely spaced parallel-runway operations is analyzed. There, only marginal capacity potential is found. This conclusion may be different for terminal areas with more favorable crosswind conditions.

Our analysis of wake vortices covers a relatively small number of wake vortices. The question of representativeness is obvious, in particular, with respect to the atmospheric conditions present during the measurements. In Fig. 16, we show histograms of crosswind and turbulent energy dissipation rates that correspond to the wake measurements. The energy dissipation rate is normalized according to $\epsilon^{*}=\left(\epsilon b_{0}\right)^{1 / 3} / w_{0}$, where $w_{0}=\Gamma_{0} / 2 \pi b_{0}$ denotes the initial descent speed. Overall, we have moderate turbulent conditions [27]. Lower turbulence levels are found for the OGE cases, consistent with the expected decrease of turbulence intensity with height in the atmospheric boundary layer. Those histograms show that a fairly broad range of conditions was sampled, and so we expect that the main conclusions will hold if we would analyze a larger data set.

A promising result of this study is that we can apply a very simple persistence model for meteorological data based on SODAR measurements to predict safe corridor clearance times using P2P.

There is still room for further optimization. For example, the resulting probability bounds of the combination P2P and SHAPE need to be assessed and adjusted to the overall user-requested probability levels. Further, the use of rectangular corridors should be replaced by elliptical corridors. Case by case, a benefit up to $40 \%$ appears possible based on simple geometric considerations.

\section{Conclusions}

In this paper, we analyze the performance of the real-time wakevortex prediction and transport model $\mathrm{P} 2 \mathrm{P}$ coupled with the simplified hazard-area prediction model SHAPE using observed (SODAR) and predicted (NOWVIV) meteorological data as input. Data from a wake and weather measurement campaign at Frankfurt airport in the fall of 2004 are used for this analysis. We employ a crosswind persistence forecast based on SODAR data. This represents a very simple method for short-term wind prediction, which is tested and compared with NOWVIV predictions up to a lead time of $6 \mathrm{~h}$. In a first step, the skill of deterministic P2P predictions using those sources of meteorological input data is assessed by a scoring procedure in which the predictions are compared with wake vortices tracked by lidar. Initially, the rms error of the deterministic NOWVIV-P2P predictions of lateral position are approximately a 

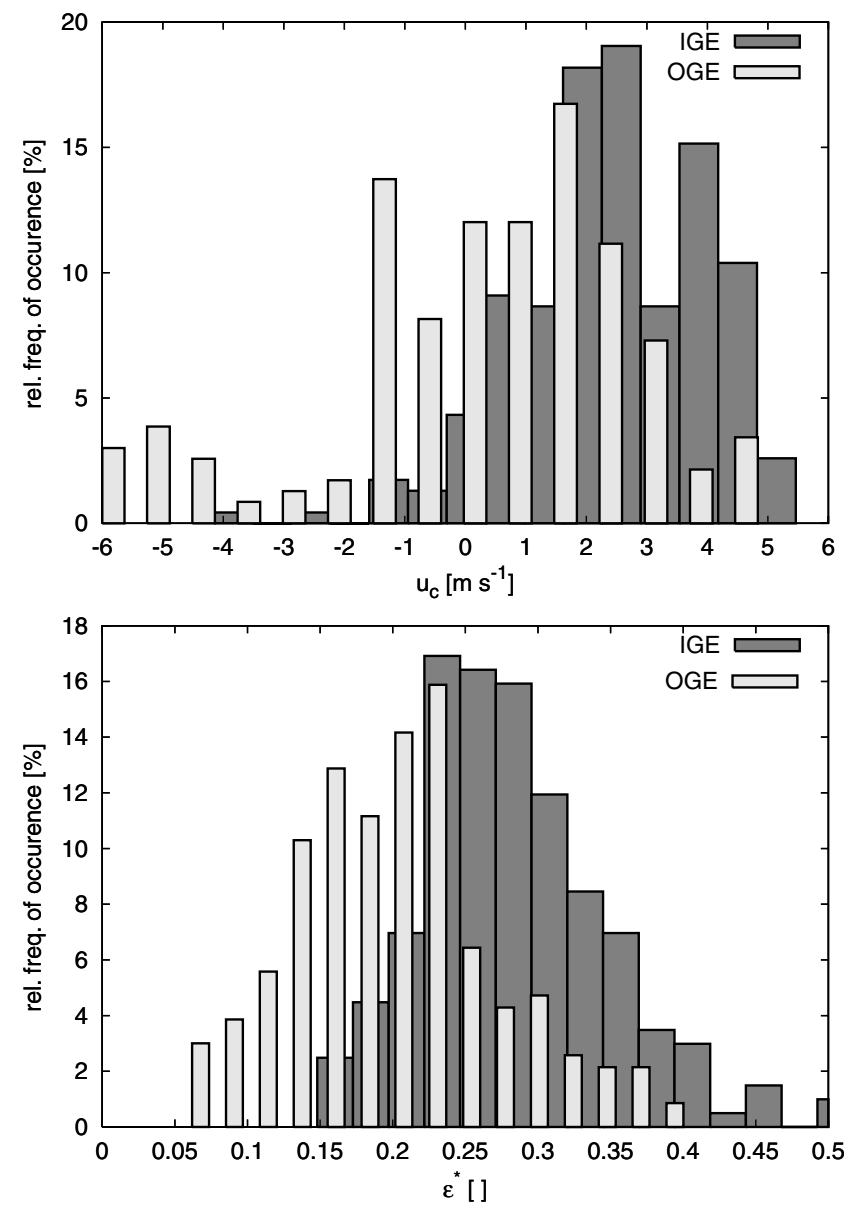

Fig. 16 Histogram of crosswind (left panel) and turbulent energy dissipation rate (right panel) corresponding to the wake-vortex measurements IGE and OGE.

factor of 2 larger than the SODAR-P2P results. We find larger SODAR-P2P prediction errors compared with NOWVIV-P2P beyond a lead time of approximately $60 \mathrm{~min}$. The same analysis for predictions OGE shows a degraded forecast performance compared with the IGE scoring results, which is due in part to larger variability of the atmospheric conditions and the missing ground proximity, which confines the wake evolution by the solid surface. Similar to the IGE results, the SODAR-P2P predictions OGE show larger errors than NOWVIV-P2P beyond a lead time of $60 \mathrm{~min}$.

We then consider $2 \sigma$ confidence levels in $\mathrm{P} 2 \mathrm{P}$ and analyze the time it takes to transport a wake laterally out of the approach corridor. We show that $\mathrm{P} 2 \mathrm{P}$ provides safe predictions IGE for both NOWVIV and SODAR input. The fraction of correct forecast is close to $100 \%$. For SODAR-P2P, this fraction starts to decrease significantly for a lead time beyond $30 \mathrm{~min}$. OGE, the fraction of correct forecast for both NOWVIV-P2P and SODAR-P2P is approximately $90 \%$. Similar to the IGE cases, this fraction starts to decrease significantly beyond a lead time of $30 \mathrm{~min}$. It is shown that the number of erroneous forecast OGE can be attributed to wakes that are rapidly transported out of the flight corridor within less than $20 \mathrm{~s}$, which appears to be irrelevant when considering typical aircraft separations. If we also consider the vertical transport out of the flight corridor for the OGE cases, the fraction of correct forecast increases. In particular, the SODAR-P2P result is more or less independent of lead time. This suggests an independence from atmospheric conditions that is related to the early stage of wake evolution in which turbulence or stratification has no pronounced effect on wake decay or rebound. During this early stage, most of the wakes are transported out of the flight corridor.

From an operational point of view, it is of interest to know if there is any capacity potential to be expected. To assure safe operations, we couple P2P with the simplified hazard-area prediction model SHAPE to compute the time to clear the corridor from hazardous wakes, because $\mathrm{P} 2 \mathrm{P}$ only predicts the probability of wake strength and position. SHAPE provides additional safety allowances to predict the dimension of the area around the wake-vortex position that has to be avoided by a follower aircraft for safe and undisturbed flight. Potential capacity gain for single-runway operations is assessed by analyzing cumulative distributions of the time to clear the flight corridor. We find that approximately $18 \%$ of the IGE wakes have left the approach corridor within $70 \mathrm{~s}$ using SODAR-P2P. For the OGE cases, we find that approximately $90 \%$ of the wakes have left the approach corridor within $70 \mathrm{~s}$. For a medium follower, approximately $90 \%$ of the wakes have left the corridor within $120 \mathrm{~s}$.

The importance of considering vertical wake transport OGE is highlighted by showing that the approach corridor is cleared from most of the vortices within $120 \mathrm{~s}$, based on NOWVIV- and SODAR$\mathrm{P} 2 \mathrm{P}$. If we consider only lateral transport, approximately $50 \%$ of the wakes remain in the approach corridor.

To conclude, based on the potential capacity gain found in this study, a possible system design could be that a wake avoidance system may rely on a numerical weather forecast along the glide path, and meteorological observations near the threshold can be used for predictions of wakes evolving in ground effect. The implementation of SODAR-P2P in an operational environment would be straightforward. From a safety point of view, the result suggests that the combination of numerical weather forecast (NOWVIV) and probabilistic wake-vortex prediction (P2P) can be used operationally for wake prediction.

To assess a realistic capacity potential through safe reduction of aircraft separations, a real traffic mix needs to be simulated. In this work, we focus on single-runway operations. The main findings presented here are also relevant for a wake avoidance system for closely spaced parallel-runway operations. The effectiveness of vertical wake transport to clear the flight corridor is expected to support the independent use of closely spaced parallel runways for landings.

\section{Acknowledgments}

We gratefully acknowledge the excellent support and work of the teams from Airbus; Deutsche Flugsicherung GmbH (DFS); DLR, German Aerospace Center; Fraport AG; and METEK during the measurement campaign. We acknowledge the fruitful cooperation with C. Schwarz and K.-U. Hahn within the DLR project Wirbelschleppe.

\section{References}

[1] Gurke, T., and Lafferton, H., "The Development of the Wake Vortices Warning System for Frankfurt Airport: Theory and Implementation," Air Traffic Control Quarterly, Vol. 5, No. 1, 1997, pp. 3-29.

[2] Hinton, D. A., Charnock, J. K., Bagwell, D. R., and Grigsby, D., "NASA Aircraft Vortex Spacing System Development Status," AIAA Paper 99-0753, Jan. 1999.

[3] Rutishauser, D. K., and O'Connor, C. J., "Aircraft Vortex Spacing System (AVOSS) Performance Update and Validation Study," NASA TM 211240, 2001.

[4] Delisi, D. P., Robins, R., Switzer, G., Lai, D., and Wang, F., "Comparison of Numerical Model Simulations and SFO Wake Vortex Wind Line Measurements," 21st AIAA Applied Aerodynamic Conference, Orlando, FL, AIAA Paper 2002-3810, 2002

[5] Hallock, J., Osgood, S., and Konopka, J., "Wake Vortex Effects on Parallel Runway Operations," 41st AIAA Aerospace Sciences Meeting and Exhibit, Reno, NV, AIAA Paper 2003-0379, 2003.

[6] Gerz, T., Holzäpfel, F., Bryant, W., Köpp, F., Frech, M., Tafferner, A., and Winckelmans, G., "Research Towards a Wake-Vortex Advisory System for Optimal Aircraft Spacing," Comptes Rendus Physique, Vol. 6, Nos. 4-5, 2005, pp. 501-523. doi:10.1016/j.crhy.2005.06.002

[7] Frech, M., and Zinner, T., "Concept of Wake Vortex Behavior Classes," Journal of Aircraft, Vol. 41, No. 3, 2004, pp. 564-570.

[8] Frech, M., Holzäpfel, F., Gerz, T., and Konopka, J., "ShortTerm Prediction of the Horizontal Wind Vector Within a Wake Vortex Warning System," Meteorological Applications, Vol. 9, No. 1, 2002, pp. 9-20. 
doi:10.1017/S1350482702001020

[9] Konopka, J., and Fischer, H., "The Wake Vortex Warning System at Frankfurt Airport," 24th Digital Avionics Systems Conference, Vol. 1, Nov. 2005, pp. 3.A.6-1-3.A.6-14.

[10] Dasey, T., and Hinton, D., "Nowcasting Requirements for the Aircraft Vortex Spacing System (AVOSS)," 8th Conference on Aviation, Range and Aerospace Meteorology, American Meteorology Society, Paper 10.1, 1999.

[11] Holzäpfel, F., Frech, M., Gerz, T., Tafferner, A., Hahn, K.-U., Schwarz, C., Joos, H.-D., Korn, B., Lenz, H., Luckner, R., and Höhne, G., "Aircraft Wake Vortex Scenarios Simulation Package-WakeScene," International Congress of Aeronautical Sciences, Paper 2006-8.6.3, 2006.

[12] Gerz, T., Tafferner, A., Rosczyk, S., Mirza, A., Turp, D., and Bot, C. L., "Improved Weather Information for Cockpit and Tower," International Congress of the Aeronautical Sciences, Paper 2006-9.5.2, 2006.

[13] Frech, M., Holzäpfel, F., Tafferner, A., and Gerz, T., "High Resolution Weather Data Base for the Terminal Area of Frankfurt Airport," Journal of Applied Meteorology and Climatology, Vol. 46, No. 11, Nov. 2007, pp. 1913-1932.

[14] Holzäpfel, F.,"Probabilistic Two-Phase Wake Vortex Decay and Transport Model," Journal of Aircraft, Vol. 40, No. 2, 2003, pp. 323-331.

[15] Holzäpfel, F., and Robins, R. E., "Probabilistic Two-Phase Aircraft Wake-Vortex Model: Application and Assessment," Journal of Aircraft, Vol. 41, No. 5, 2004, pp. 1117-1126.

[16] Holzäpfel, F., "Probabilistic Two-Phase Aircraft Wake-Vortex Model: Further Development and Assessment," Journal of Aircraft, Vol. 43, No. 3, 2006, pp. 700-708.

[17] Holzäpfel, F., and Steen, M., "Aircraft Wake-Vortex Evolution in Ground Proximity: Analysis and Parameterization," AIAA Journal, Vol. 45, No. 1, 2007, pp. 218-227. doi:10.2514/1.23917

[18] Hahn, K.-U., Schwarz, C., and Friehmelt, H., "A Simplified Hazard Area Prediction (SHAPe) Model for Wake Vortex Encounter Avoidance," 24th International Congress of the Aeronautical Sciences (ICAS 2004), edited by P. I. Grant, Optimage, Edinburgh, Scotland, U.K., and International Congress of the Aeronautical Sciences,
Stockholm, Sweden, 2004.

[19] Schwarz, C. W., and Hahn, K.-U., "Full-Flight Simulator Study for Wake Vortex Hazard Area Investigation," Aerospace Science and Technology, Vol. 10, No. 2, 2006, pp. 136-143. doi:10.1016/j.ast.2005.09.005

[20] Grell, G., Dudhia, J., and Stauffer, D. R., "A Description of the FifthGeneration Penn State/NCAR Mesoscale Model (MM5)," National Center for Atmospheric Research NCAR/TN-397+IA, Boulder, CO, 1994.

[21] Grell, G., Schade, L., Knoche, R., Pfeiffer, A., and Egger, J., "Nonhydrostatic Climate Simulations of Precipitation over Complex Terrain," Journal of Geophysical Research, Vol. 105, No. D24, 2000, pp. 29595-29608. doi:10.1029/2000JD900445

[22] Steppeler, J., Doms, G., Schättler, U., Bitzer, H. W., Gassmann, A., Damrath, U., and Gregoric, G., "Meso-Gamma Scale Forecasts Using the Non-Hydrostatic Model LM," Meteorology and Atmospheric Physics, Vol. 82, Nos. 1-4, 2003, pp. 75-96. doi:10.1007/s00703-001-0592-9

[23] Köpp, F., Rahm, S., and Smalikho, I., "Characterization of Aircraft Wake Vortices by $2 \mathrm{~m}$ Pulsed Doppler Lidar," Journal of Atmospheric and Oceanic Technology, Vol. 21, No. 2, 2004, pp. 194-206. doi:10.1175/1520-0426(2004)021<0194:COAWVB >2.0.CO;2

[24] Köpp, F., Rahm, S., Smalikho, I., Dolfi, A., Cariou, J.-P., Harris, M., and Young, R. I., "Comparison of Wake-Vortex Parameters Measured by Pulsed and Continuous-Wave Lidars," Journal of Aircraft, Vol. 42, No. 4, 2005, pp. 916-923.

[25] Nurmi, P., "Recommendation on the Verification of Local Weather Forecasts," European Centre for Medium-Range Weather Forecasts, TM 430, Reading, England, U.K., 2003.

[26] Frauenkron, H., Biegholdt, J., Maiss, M., Nalpanis, P., and Smith, E., "FLIP: Flight Performance Using Frankfurt ILS. A Statistical Evaluation of Navigational Performance of ILS-Approaches at Frankfurt Airport," DFS, German Air Navigation Services, Offenbach, Germany, 2001.

[27] Sarpkaya, T., "New Model for Vortex Decay in the Atmosphere," Journal of Aircraft, Vol. 37, No. 1, 2000, pp. 53-61. 